Original

\title{
Afectación de la calidad de vida relacionada con la salud en mujeres con Incontinencia Urinaria Mixta
}

\author{
Pere Arañó*, Pablo Rebollo**, Diego González-Segura Alsina*** \\ *Unidad de Urología funcional y femenina. Servicio de Urología. Fundació Puigvert. Barcelona. \\ **BAP Health Outcomes Research, S.L. Oviedo. \\ ***Departamento Médico, Laboratorios Almirall S.A. Barcelona.
}

\begin{abstract}
Resumen
Introducción: $\mathrm{El}$ análisis de la calidad de vida relacionada con la salud (CVRS) en mujeres con Incontinencia Urinaria (IU) Mixta puede ser un indicador útil en el manejo del tratamiento precoz orientado al tipo de IU que domine: de esfuerzo o de urgencia. El objetivo del presente estudio fue evaluar la CVRS de mujeres con IU Mixta, comparando el impacto según síntomas de IU de urgencia o de esfuerzo.

Material y métodos: Estudio epidemiológico, transversal y multicéntrico, en el que se evaluó la CVRS en 1.100 mujeres con síntomas de IUM. Para evaluar la CVRS se utilizó la versión española del cuestionario "King's Health Questionnaire" (KHQ). Además se recogieron datos personales de las pacientes, datos sobre antecedentes patológicos y datos clínicos relacionados con el diagnóstico y tratamiento de la IU.

Resultados: Las infecciones urinarias fueron el antecedente patológico más frecuente. El tiempo medio transcurrido desde el inicio de los síntomas de IU fue de 4 años y el $44 \%$ de las mujeres había ya recibido algún tratamiento para la IU. Se observó un mayor uso de compresas $(63,9 \%)$ que de salva-slips $(36 \%)$ y de pañales $(11,2 \%)$. La puntuación global media en el KHQ fue de 42,2 (D.E.=18,7); el mayor impacto en la CVRS correspondió a las dimensiones: Impacto de la IU, Afectación por Problemas Urinarios, y Limitaciones Físicas. La afectación de la CVRS por el síntoma de IU de Urgencia fué mayor que por el síntoma de IU de Esfuerzo ( $<<0,0001)$. En el modelo de regresión para la puntuación global del KHQ, las variables que mostraron mayor asociación, además de las ya mencionadas, fueron el prolapso genital y el síntoma de IU en el acto sexual.

Conclusión: Existe un importante deterioro en la CVRS de las mujeres con IU Mixta, estando este impacto fuertemente asociado a la presencia de síntomas de IU de Urgencia.
\end{abstract}

Palabras clave: Incontinencia urinaria. Mujeres. Calidad de vida. Incontinencia urinaria mixta.

Assessment of the Health-Related Quality of Life impact in female with Mixed Urinary Incontinence.

Abstract

Background: Health-related quality of life (HRGoL) assessment on female with urinary incontinence (UI) may be a useful indicator in the management of early treatment depending on the sort of UI which is dominant: Stress UI or Urge UI. The objective of the present study was to evaluate the HRQoL of female with mixed UI, comparing the impact of symptoms of Urge UI and Stress UI.

Patients and methods: This is an epidemiologic, cross-sectional and multicenter study. HRQoL of 1,100 women with mixed UI was evaluated using the Spanish version of "King's Health Questionnaire" (KHQ). Personal data of patients were also collected along with medical records and clinical data related to diagnostic and treatment of UI.

Results: Urinary infection was the most frequent medical record found. Mean time since start of UI symptoms was 4 years and $44 \%$ of patients had been received some treatment for UI. It was observed a higher use of sanitary napkins (63.9\%) than pantyliners (36\%) and diapers (11.2\%). Mean global score of the KHQ was 42.2 (S.D.= 18.7); higher impact on HRQoL was shown in dimensions: UI impact, Urinary problems and Physical Limitations. Urge UI symptoms cause higher impact on HRQoL than Stress UI symptoms ( $\mathrm{p}<0.0001$ ). The logistic regression model performed for global KHQ score showed that variables strongly associated were Urge UI, genital prolapse and coital UI symptom.

Conclusions: Women with mixed UI showed and important impact on HRQoL. This impact is strongly associated with presence of symptoms of Urge UI.

Keywords: Urinary incontinence. Female. Quality of life. Mixed urinary incontinence. 
$\mathrm{L}^{2}$ a incontinencia urinaria (IU) se define como la pérdida involuntaria de orina objetivamente demostrable que origina un problema social o higiénico $^{1}$. Incluye, por tanto, en su propia definición la necesaria afectación de la calidad de vida relacionada con la salud (CVRS) y por ello este aspecto ha sido a menudo estudiado en las personas que la padecen. Es una patología muy prevalente en nuestro país: se estima que padecen IU entre el 25 y el $45 \%$ de las mujeres $^{2}$ y entre el 7 y el $11 \%$ de los varones, mayores de 20 años. A pesar de que la incontinencia no implica un pronóstico de gravedad, limita la autonomía, reduce la autoestima y deteriora sensiblemente la $\mathrm{CVRS}^{3}$.

La "International Continence Society" (ICS), en su documento sobre estandarización de terminología, establece las definiciones de los diferentes tipos de IU. La IU Mixta (IUM) es definida como la molestia derivada de la pérdida involuntaria de orina asociada a urgencia y también a esfuerzo ${ }^{4}$. Aunque tanto la prevalencia global de IU como la distribución de sus diferentes tipos son cifras que varían mucho en los estudios publicados, dependiendo de la definición empleada y del método de selección de la muestra estudiada, en un estudio europeo que incluyó datos de nuestro país, del total de mujeres mayores de 18 años con incontinencia urinaria, el $33 \%$ expresaron síntomas que sugerían la presencia de IUM $^{5}$. Según diferentes estudios, la prevalencia de la IUM se sitúa cerca del 50\% y aumenta con la $\operatorname{edad}^{6}$.

Para medir la CVRS en de las personas que padecen IU se han utilizado cuestionarios genéricos, como el Cuestionario de Salud SF-36 y sus versiones reducidas, pero también específicos de IU, como el $\mathrm{I}_{-} \mathrm{QOL}^{7}$, el Bristol Female Lower Urinary Tract Symptoms Questionnaire ${ }^{8}$ y el King's Health Questionnaire $(\mathrm{KHQ})^{9}$ del que se dispone de una versión española adecuadamente adaptada y validada en nuestro país ${ }^{10}$. De acuerdo a estudios publicados, parece que el impacto en la CVRS sería mayor en el caso de la IU de Urgencia (IUU) que en el de la IU de Esfuerzo (IUE) ${ }^{11}$. Los síntomas de urgencia afectan especialmente la movilidad y por ello la IUU se asocia a sentimientos de malestar y preocupación, aunque en todo caso, ambos tipos de IU reducen la $\mathrm{CVRS}^{7}$.
La afectación de la CVRS puede ser un indicador útil para decidir la instauración de un tratamiento precoz de la IUM, orientándolo al tipo que predomine: IU de Esfuerzo o IU de Urgencia.

Con el objetivo principal de evaluar el impacto de la IU Mixta (IUM) en la calidad de vida de las mujeres que la padecen, se realizó el estudio QoLIUM ("Guality of Life" medida mediante el King's Health Questionnaire, en Incontinencia Urinaria Mixta). Este estudio también perseguía comparar el impacto de la IUM en la CVRS de las mujeres según predominancia de síntomas de urgencia o de esfuerzo.

\section{SUJETOS Y MÉTODO}

Estudio epidemiológico, transversal y multicéntrico de evaluación de la CVRS en mujeres con síntomas de IUM, definida como presencia de pérdida involuntaria de orina asociada tanto a la urgencia como al esfuerzo. En el estudio participaron 1.448 mujeres reclutadas en 197 centros de urología y ginecología de nuestro país, por 275 investigadores. De estas mujeres 348 fueron excluidas del presente análisis por incumplir uno o más de los criterios de inclusión en el estudio (Tabla 1). Se analizaron los datos de las restantes 1.100 mujeres.

Para evaluar la CVRS se utilizó el cuestionario "King's Health Questionnaire" ${ }^{9}$ en su versión española adecuadamente validada ${ }^{10}$. El KHQ consta de 21 ítems distribuidos en 9 dimensiones: percepción del estado de salud general ( 1 ítem); afectación de la IU (1 item), limitaciones en sus actividades diarias ( 2 items); limitaciones físicas ( 2 ítems); limitaciones sociales (2 ítems); relaciones personales ( 3 ítems); emociones (3 ítems); sueño/energía (2 ítems) e impacto de la incontinencia (5 ítems). Cada ítem, exceptuando el primero, tiene una escala de respuesta con 4 posibles opcio-
Tabla 1. Pacientes valorables

\begin{tabular}{lcc}
\hline Pacientes valorables & $\mathbf{N}$ & $\mathbf{\%}^{\mathbf{1}}$ \\
\hline Total de casos reclutados & 1.448 & 100,00 \\
Valorables & 1.100 & 75,97 \\
No valorables $^{2}$ & 348 & 24,03 \\
$\quad$ No presenta IUE & 240 & 16,57 \\
$\quad$ No presenta IUU & 148 & 10,22 \\
$\quad$ Pérdidas de orina con 4 o menos meses de evolución & 33 & 2,28 \\
$\quad$ Alguna patología o condición excluyente & 78 & 5,39 \\
$\quad$ Todo el cuestionario KHQ vacío & 4 & 0,28 \\
\hline
\end{tabular}

${ }^{1}$ Porcentaje calculado sobre el total de pacientes reclutadas ( $N=1448$ )

${ }^{2}$ Una paciente podía ser considerada no valorable por más de un motivo simultáneamente. 
nes. El rango de puntuaciones de cada dimensión va de 0 (mejor CVRS) a 100 (peor CVRS) y se calcula mediante la puntuación media de los ítems que forman la dimensión. Este cuestionario consta además de una serie de 11 preguntas en las que se interroga a la paciente sobre el impacto que causan los síntomas urinarios.

Además se recogieron datos antropométricos, antecedentes patológicos y datos clínicos relacionados con el diagnóstico y tratamiento de la IU (tiempo desde el inicio de los síntomas, consultas previas por IU con profesionales sanitarios, tratamientos previos de la IU, presencia de aumento de la frecuencia miccional diaria y de nicturia, presencia de prolapso genital de grado III o superior, incontinencia fecal, estreñimiento, número de embarazos a término y tratamiento hormonal sustitutivo).

\section{ANÁLISIS ESTADÍSTICO}

El tratamiento estadístico de los datos se efectuó con el paquete estadístico SPSS versión 13. La variable de interés fue la Calidad de Vida Relacionada con la Salud de los pacientes, evaluada con la versión española del cuestionario KHQ. Los ítems de la variable principal se describieron a través de tablas de frecuencia absoluta y relativa. Adicionalmente se describieron las 9 dimensiones que forman este cuestionario así como la puntuación total del mismo. La comparación de las dimensiones del test, así como de la puntuación total, según el tipo de IU que presenten los pacientes, se realizó mediante el test de Kruskal-Wallis.

Mediante un modelo de regresión lineal se analizaron los factores presentados por las pacientes con incontinencia urinaria mixta (características sociodemográficas, sintomas urinarios, tiempo de evolución de la IU, ...) influyentes en la calidad de vida de la paciente (puntuación global de cuestionario KHQ).

Las variables continuas se describieron utilizando medidas de tendencia central (media, mediana) y medidas de dispersión (desviación estándar, mínimo y máximo). Las variables categóricas se describieron a través de tablas de frecuencia absoluta y relativa.

La comparación de variables continuas entre grupos de pacientes se realizó mediante el test no paramétrico de Mann-Whitney, y en caso de ser más de 2 grupos se utilizó el test no paramétrico de KruskalWallis. La comparación de variables categóricas se realizarán con la prueba estadística Chi-cuadrado.

Todas las pruebas estadísticas fueron bilaterales y se efectuaron con un nivel de significación del 0,05.

\section{RESULTADOS}

La descripción de la muestra estudiada se presenta en la Tabla 2. Las infecciones urinarias fueron el antecedente patológico más frecuente en las mujeres estudiadas, seguido de las enfermedades digestivas y de la cirugía abdominal previa. Las características clínicas de la IU se presentan en la Tabla 3. El tiempo medio transcurrido desde el inicio de los síntomas de IU fue de algo más de 4 años y el $44 \%$ de las mujeres había ya recibido algún tratamiento para la IU, siendo el más frecuente entre ellos el farmacológico. Los síntomas de frecuencia y nicturia se presentaron en más de las 2/3 partes de la muestra estudiada.

Las puntuaciones medias en las dimensiones del cuestionario KHQ se presentan en la Figura 1. Como se puede observar en la figura, las puntuaciones más altas (mayor impacto en la CVRS) corresponden a las dimensiones Impacto de la IUImIU, Afectación por Problemas Urinarios-APU y Limitaciones Físicas-LF. La frecuencia de presenta-

Tabla 2. Descripción de la muestra estudiada $(\mathrm{N}=1.100)$

Edad: media (D.E.)

$57,2(12,5)$

IMC: media (D.E.)

$26,2(4,0)$

$\mathrm{N}^{\circ}$ de hijos: media (D.E.)

$2,7(1,2)$

Menopausia: $n$ (\%)

$732(66,5)$

Infecciones urinarias: $\mathrm{n}(\%)$

$483(43,9)$

$\mathrm{N}^{\circ}$ inf. urinarias: media episodios/año (D.E.) $\quad 2,9(2,2)$

Enfermedades digestivas: $\mathrm{n}(\%)$

$179(16,3)$

Enfermedades cardiovasculares: n (\%)

$96(8,7)$

Intervención quirúrgica digestiva: $\mathrm{n}(\%)$

$130(11,8)$

Otra cirugía abdominal: $\mathrm{n}(\%)$

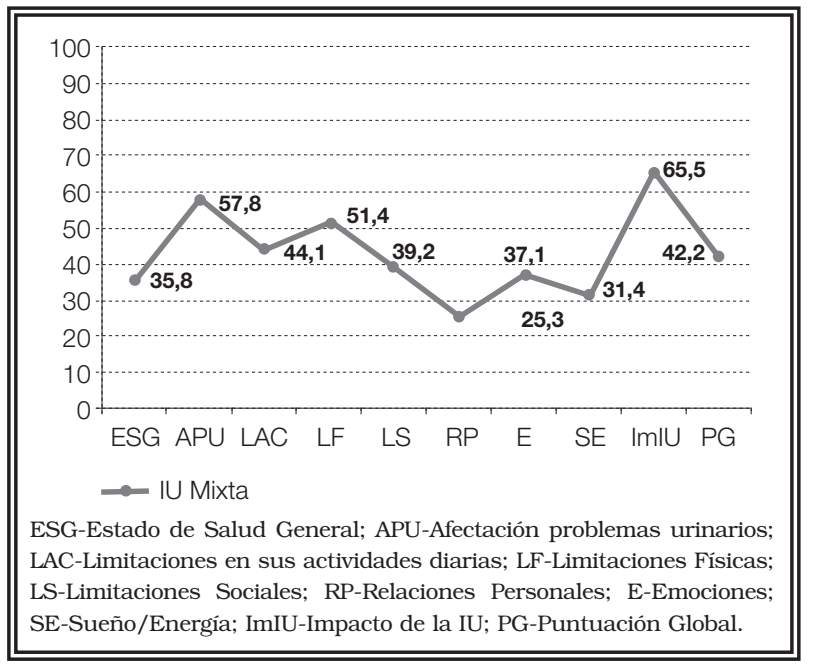

FIGURA 1. Puntuaciones medias en las dimensiones del cuestionario KHQ (N=1.100). 
Tabla 3. Características clínicas de la incontinencia urinaria

Años desde inicio síntomas $\left(\mathrm{N}=1.088^{1}\right)$ : media (D.E.)

Tratamientos para la IU $(\mathrm{N}=487)$ : n (\%)

Farmacológico

Reeducación del suelo pélvico

Quirúrgico

Otros tratamientos

Uso de absorbentes $/ 24$ horas $(\mathrm{N}=1.100)$

$\mathrm{N}^{\mathrm{o}}$ de salva-slips $(\mathrm{n}=396)$ : media (D.E.)

$\mathrm{N}^{\circ}$ de compresas $(\mathrm{n}=703)$ : media (D.E)

$\mathrm{N}^{\mathrm{o}}$ de pañales $(\mathrm{n}=123)$ : media (D.E.)

Síntoma de frecuencia aumentada $(\mathrm{N}=1.100)$ : $\mathrm{n}(\%)$

Síntoma de nicturia $(\mathrm{N}=1.100)$ : $\mathrm{n}(\%)$

Prolapso genital grado III o superior $(\mathrm{N}=1.100)$ : $\mathrm{n}(\%)$

Incontinencia fecal $(\mathrm{N}=1.100)$ : $\mathrm{n}(\%)$

Estreñimiento $(\mathrm{N}=1.100): \mathrm{n}(\%)$

Tratamiento hormonal sustitutivo actual $(\mathrm{N}=1.100)$ : $\mathrm{n}(\%)$

${ }^{1}$ Número de pacientes con dato "Años desde inicio síntomas" valorable.

ción de los diferentes síntomas urinarios y el grado de afectación que producen, según los ítems específicos del cuestionario $\mathrm{KHQ}$, se presentan en la Tabla 4, en la que puede observarse que la afectación por el síntoma de IU de Urgencia es mayor que por el síntoma de IU de Esfuerzo siendo esta diferencia estadísticamente significativa $(\mathrm{p}<0,0001)$.

Las puntuaciones del cuestionario KHQ resultaron ser mayores, cuanto mayor es la afectación por la presencia del síntoma de IU de Urgencia (Fig. 2) o de IU de Esfuerzo (Fig. 3) siendo en ambos casos las diferencias entre los grupos estadísticamente significativas $(\mathrm{p}<0,0001)$.

El análisis univariado de asociaciones entre variables de las pacientes y la puntuación global obtenida en el KHQ, se presenta en la Tabla 5. El
$4,4(4,8)$

modelo de regresión lineal construido con aquellas variables que habían mostrado una asociación estadísticamente significativa con la puntuación global del KHQ en el análisis univariado, se presenta en la Tabla 6. Se realizó una comparación entre la puntuación del KHQ de los síntomas de IU de urgencia y los sintomas de IU de esfuerzo, y sus grados de afectación. Se observó que el hecho de presentar síntomas de IU de Urgencia con mucha afectación, aumentaba la puntuación en el KHQ en 17,58 puntos, mientras que el grado moderado, aumentaba la puntuación en 7,69 puntos. En el caso del síntoma de IU de Esfuerzo, en cambio, el hecho de presentar mucha afectación aumentaba la puntuación en el KHQ en 8 puntos, mientras que el grado moderado, la aumentaba en sólo 1,79 puntos.

\section{DISCUSIÓN}

De acuerdo a los resultados del presente estudio las pacientes con IU Mixta muestran un importante impacto en su calidad de vida, influyendo en mayor medida en este impacto (más del doble) los síntomas de IU de Urgencia que los de IU de Esfuerzo.

El impacto en la calidad de vida, de acuerdo a la puntuación global en el cuestionario $\mathrm{KHQ}$, fue superior en la muestra de mujeres con síntomas de IU Mixta del presente estudio $(42,2$; D.E. $=18,7)$ que en la de otros estudios publicados realizados en mujeres con sintomas de vejiga Hiperactiva $(38,3 \text {; D.E. }=19,2)^{12}$ o con sintomas de IU $(39,3 ; \text { S.E. }=22,11)^{13}$. Estas dife-

Tabla 4. Síntomas urinarios y grado de afectación según los ítems específicos del cuestionario KHQ

\begin{tabular}{lcccc}
\hline Síntoma & Incidencia & Un poco & Moderadamente & Mucho \\
\hline Frecuencia & $1.040(94,5 \%)$ & $242(23,3 \%)$ & $500(48,1 \%)$ & $298(28,7 \%)$ \\
Nicturia & $918(83,5 \%)$ & $362(39,4 \%)$ & $392(42,7 \%)$ & $164(17,9 \%)$ \\
IU por Urgencia & $1.100(100 \%)$ & $248(22,5 \%)$ & $512(45,5 \%)$ & $340(30,9 \%)$ \\
IU por Esfuerzo & $1.100(100 \%)$ & $401(36,5 \%)$ & $474(43,1 \%)$ & $225(20,5 \%)$ \\
Eneuresis nocturna & $484(44 \%)$ & $277(57,2 \%)$ & $145(30,0 \%)$ & $62(12,8 \%)$ \\
IU en acto sexual & $423(38,5 \%)$ & $269(63,6 \%)$ & $103(24,3 \%)$ & $51(12,1 \%)$ \\
Infecciones en vías urinarias & $441(40,1 \%)$ & $259(58,7 \%)$ & $147(33,3 \%)$ & $35(7,9 \%)$ \\
Dolor en vejiga & $399(36,3 \%)$ & $293(73,4 \%)$ & $91(22,8 \%)$ & $15(3,8 \%)$ \\
Dificultad al orinar & $292(26,5 \%)$ & $238(81,5 \%)$ & $48(16,4 \%)$ & $6(2,1 \%)$ \\
Otros problemas urinarios & $76(6,9 \%)$ & $61(80,3 \%)$ & $13(17,1 \%)$ & $2(2,6 \%)$ \\
\hline
\end{tabular}




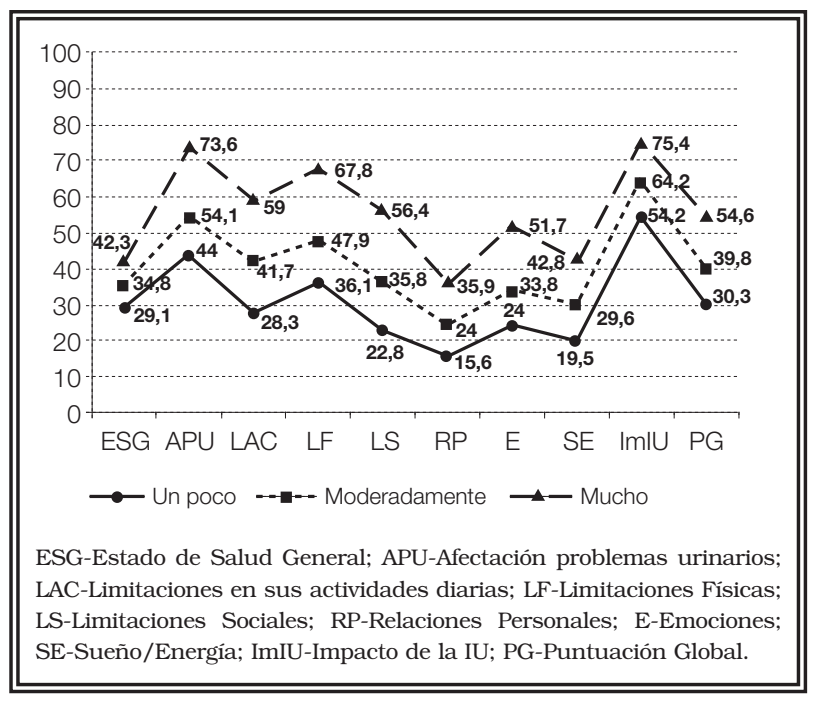

FIGURA 2. Puntuaciones medias en las dimensiones del cuestionario $\mathrm{KHQ}(\mathrm{N}=1.100)$ según el grado de afectación por el síntoma de IU de Urgencia.

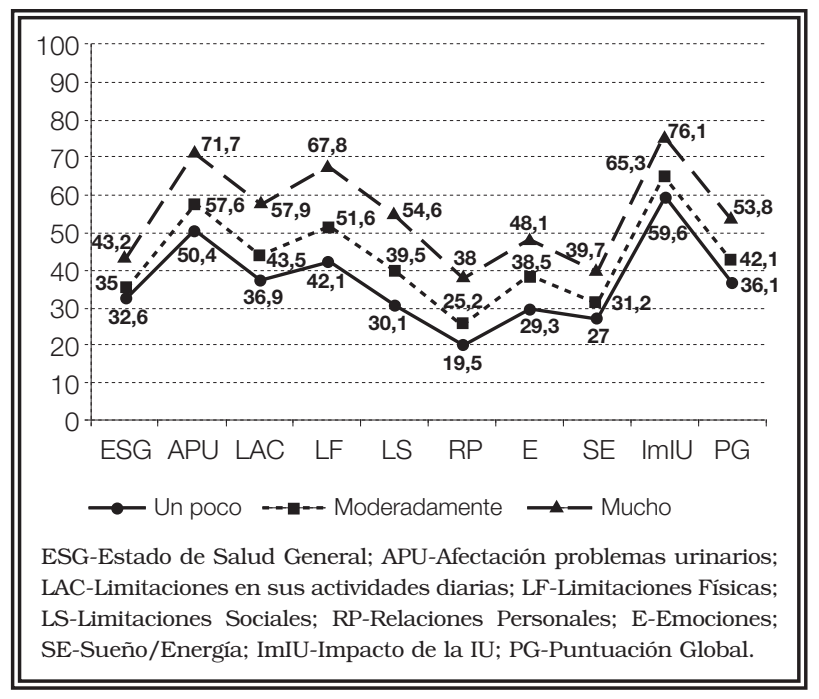

FIGURA 3. Puntuaciones medias en las dimensiones del cuestionario $\mathrm{KHQ}(\mathrm{N}=1.100)$ según el grado de afectación por el sintoma de IU de Esfuerzo.

Tabla 5. Análisis de asociaciones entre variables de las pacientes y puntuación global del KHQ.

\section{Puntuación del cuestionario KHQ: media (D.E.)}

\begin{tabular}{|c|c|c|c|c|}
\hline & Menos de 2 & Entre 2 y 4 & Más de 4 & $\mathbf{p}$ \\
\hline $\mathrm{N}^{\circ}$ salva-slip /24 horas & $30,7(16,9)$ & $37,9(17,6)$ & $43,6(17,8)$ & 0,0005 \\
\hline $\mathrm{N}^{\circ}$ compresas /24 horas & $37,7(18,2)$ & $44,8(16,7)$ & $54,2(18,1)$ & $<0,0001$ \\
\hline \multirow[t]{2}{*}{$\mathrm{N}^{\circ}$ pañales / 24 horas } & $48,0(16,9)$ & $58,1(18,0)$ & $57,8(13,7)$ & 0,0209 \\
\hline & No & Si & & \\
\hline Antecedente de infección urinaria & $40,3(18,7)$ & $44,8(18,3)$ & 0,0001 & \\
\hline Antecedente de enfermedad digestiva & $41,5(18,3)$ & $46,1(20,0)$ & 0,0048 & \\
\hline Antecedente de enfermedad cardiovascular & $41,8(18,5)$ & $47,2(19,7)$ & 0,0169 & \\
\hline Antecedente de intervención quirúrgica digestiva & $41,6(18,6)$ & $47,1(18,5)$ & 0,0013 & \\
\hline Antecedente de otra cirugía abdominal & $41,3(18,4)$ & $45,7(19,3)$ & 0,0027 & \\
\hline Prolapso genital grado III o superior & $41,1(18,3)$ & $53,7(18,2)$ & $<0,0001$ & \\
\hline Incontinencia fecal & $42,1(18,6)$ & $50,7(18,9)$ & 0,0357 & \\
\hline Menopausia & $39,2(19,2)$ & $43,8(18,2)$ & 0,0001 & \\
\hline Síntoma frecuencia & $33,1(15,8)$ & $42,8(18,7)$ & 0,0001 & \\
\hline Síntoma nicturia & $33,0(17,1)$ & $44,1(18,4)$ & $<0,0001$ & \\
\hline Síntoma Eneuresis nocturna & $37,2(16,8)$ & $48,7(19,0)$ & $<0,0001$ & \\
\hline Síntoma IU en acto sexual & $39,1(17,7)$ & $47,0(19,1)$ & $<0,0001$ & \\
\hline Síntoma infecciones en vías urinarias & $39,6(18,2)$ & $46,1(18,7)$ & $<0,0001$ & \\
\hline Síntoma dolor en vejiga & $39,5(17,7)$ & $47,0(19,4)$ & $<0,0001$ & \\
\hline \multirow[t]{2}{*}{ Síntoma dificultad al orinar } & $40,6(18,0)$ & $46,6(19,8)$ & $<0,0001$ & \\
\hline & Un poco & Moderadamente & Mucho & \\
\hline Síntoma IU por Urgencia & $30,3(14,6)$ & $39,8(16,0)$ & $54,6(18,1)$ & $<0,0001$ \\
\hline Síntoma IU por Esfuerzo & $36,1(17,9)$ & $42,1(17,2)$ & $53,8(17,7)$ & $<0,0001$ \\
\hline
\end{tabular}

rencias se centran fundamentalmente en las puntuaciones de las dimensiones Impacto de la IUImIU, Relaciones Personales-RP, Limitaciones Sociales-LS, Limitaciones Físicas-LF y Limitaciones en las Actividades Cotidianas-LAC, que son superiores en la muestra del presente estudio respecto a las descripciones que se hacen en los otros dos antes citados. 
Tabla 6. Modelo de Regresión Lineal Múltiple para la puntuación global del cuestionario KHQ

\begin{tabular}{lccccc}
\hline & B & E.T. & B estandarizado & t & p \\
\hline Constante & 13,01 & 3,30 & - & 3,94 & 0,0001 \\
Nicturia & 4,72 & 1,06 & 0,12 & 4,43 & $<0,0001$ \\
IMC & 0,37 & 0,12 & 0,08 & 2,99 & 0,0029 \\
Años desde inicio sintomas IU & 0,24 & 0,11 & 0,06 & 2,16 & 0,0308 \\
Intervención qca. Digestiva & 4,27 & 1,47 & 0,07 & 2,90 & 0,0038 \\
Prolapso genital $\geq$ grado III & 6,35 & 1,70 & 0,10 & 3,75 & 0,0002 \\
Estreñimiento & $-2,27$ & 0,96 & $-0,06$ & $-2,36$ & 0,0184 \\
IUU moderada afectación & 7,69 & 1,25 & 0,21 & 6,15 & $<0,0001$ \\
IUU mucha afectación & 17,58 & 1,43 & 0,44 & 12,32 & $<0,0001$ \\
IUE moderada afectación & 1,79 & 1,11 & 0,05 & 1,62 & 0,1063 \\
IUE mucha afectación & 8,00 & 1,43 & 0,17 & 5,58 & $<0,0001$ \\
Eneuresis nocturna & 4,49 & 1,09 & 0,12 & 4,10 & $<0,0001$ \\
IU en el acto sexual & 4,77 & 1,08 & 0,13 & 4,40 & $<0,0001$ \\
\hline
\end{tabular}

El tiempo desde el inicio es muy largo (algo más de 4 años) y aunque con poca fuerza, es una variable que mostró asociación independiente con la CVRS medida por la puntuación global del KHQ. Con tan larga evolución se entiende que casi la mitad de las mujeres ya habían recibido algún tratamiento para la IU, más frecuentemente farmacológico, aunque no se evidenciaron diferencias en la puntuación global del KHQ según el tratamiento recibido (farmacológico, reeducación o ambos).

La afectación de la CVRS por la presencia del síntoma de IU de Urgencia es valorado por las pacientes como mayor que por el síntoma de IU de Esfuerzo, estando ambos síntomas asociados a mayores puntuaciones en el KHQ (peor CVRS) cuanto mayor sea el grado de afectación. En conclusión los resultados presentados confirman el considerable deterioro que la IU Mixta produce en la calidad de vida relacionada con la salud de la mujer que la padece, estando este impacto asociado, en más del doble de intensidad, a la presencia de síntomas de IU de Urgencia que de IU de Esfuerzo. Resolver o aliviar los síntomas de IU de Urgencia en este grupo de mujeres, permitiría lograr una sustancial mejoría en su calidad de vida.

\section{Agradecimientos}

Agradezco la colaboración de todos los médicos urólogos y ginecólogos que han participado en el estudio GoLIUM, por proporcionar los datos necesarios para su realización, así como a Laboratorios Almirall SA, cuya colaboración no condicionada ha permitido que este estudio pudiera llevarse a cabo.

\section{REFERENCIAS}

1. Abrams P, Cardozo L, Fall M, Griffiths D, Rosier P, Ulmsten U, et al. The standardisation of terminology in lower urinary tract function report from the standardisation sub-committee of the International Continence Society. Urology 2003; 61(1): 37-49.

2. Revista Española de Economía de la Salud. La enfermedad silenciosa. Rev Es 2002; 1 (2): 27-30.

3. Espuña Pons M. Incontinencia de orina en la mujer. Med Clin (Barc) 2003 Apr 5;120(12):464-72.

4. Ulmsten U. Some reflections and hypotheses on the pathophysiology of female urinary incontinence. Acta Obstet Gynecol Scand Suppl. 1997; 166:3-8.

5. Hunskaar S, Lose G, Sykes D, Voss S. The prevalence of urinary incontinence in women in four European countries. BJU Int. 2004 Feb;93(3):324-330.

6. Hampel C, Wienhold D, Benken N, Eggersmann C, Thuroff JW. Prevalence and natural history of female incontinence. Eur Urol 1997;32 Suppl 2:3-12.

7. Wagner TH, Patrick DL, Bavemdam TG, Martin ML, Buesching DP. Quality of life of persons with urinary incontinence: development of a new measure. Urology. 1996 Jan;47(1):67-71; discussion 71-72.

8. Jackson S, Donovan J, Brookes S, Eskford S, Swithinbank L, Abrams P. The Bristol Female Lower Urinary Tract Symptoms questionnaire: development and psychometric testing. Br J Urol. 1996 Jun;77(6):805-812.

9. Kelleher CJ, Cardozo LD, Khullar V, Salvatore S. A new questionnaire to assess the quality of life of urinary incontinent women. Br J Obstet Gynaecol. 1997 Dec;104(12):1374-1379.

10. Badia X; Castro D; Conejero J. Validez del cuestionario King's Health para la evaluación de la calidad de vida en pacientes con incontinencia urinaria. Med Clin (Barc) 2000; 114: 647-652.

11. Hunskaar S, Vinsnes A. The quality of life in women with urinary incontinence as measured by the sickness impact profile. J Am Geriatr Soc. 1991 Apr;39(4):378-382.

12. Espuña M, Puig M. Síntomas del tracto urinario inferior en la mujer y afectación de la calidad de vida. Resultados de la aplicación del King’s Health Questionnaire. Actas Urol Esp. 2006 Jul-Aug;30(7):684-691.

13. Espuña M, Castro D, Carbonell C, Dilla T. Comparación entre el cuestionario ICIQ-UI Short Form y el King's Health Questionnaire como instrumentos de evaluación de la incontinencia urinaria en mujeres. Actas Urol Esp. 2007 May;31(5):502-510.

Correspondencia autor: Dr. Pablo Rebollo

BAP Health Outcomes Research. Pg. Ind. Espíritu Santo,

Parcela 37, $1^{\circ} \mathrm{B}$ - 33010 Oviedo. Tel.: 985793704

E-mail autor: pablo@baphealth.com

Información artículo: Original - Incontinencia

Trabajo recibido: septiembre 2008

Trabajo aceptado: octubre 2008 